\title{
Relativistic corrections in displacement measuring interferometry
}

\author{
Ralf K. Heilmann, ${ }^{\text {a) }}$ Paul T. Konkola, Carl G. Chen, and Mark L. Schattenburg \\ Space Nanotechnology Laboratory, Center for Space Research, Massachusetts Institute of Technology, \\ Cambridge, Massachusetts 02139
}

(Received 1 June 2000; accepted 26 July 2000)

\begin{abstract}
Displacement measuring interferometry is based on measuring the Doppler frequency shift that a beam of radiation undergoes upon reflection off a mirror connected to a moving stage. Usually the velocity of the reflecting stage is very small compared to the speed of light and is therefore deduced using the classical expression for the Doppler shift. We calculate relativistic corrections to the Doppler frequency shift, considering arbitrary stage motion in two dimensions and multiple passes through the moving interferometer arm. Changes in optical path lengths due to the varying stage displacement are explicitly taken into account. For stage velocities on the order of only $1 \mathrm{~m} / \mathrm{s}$ the resulting corrections to the classically derived stage displacement can amount to nanometers. We discuss model velocity profiles similar to those currently employed in industrial step-and-scan systems for integrated circuit manufacturing, and for recently proposed scanning-beam interference lithography schemes. Expected future increases in stage speed and wafer sizes will necessitate the inclusion of relativistic corrections to the Doppler shift to maintain pattern placement accuracy at the nanometer level. (C) 2000 American Vacuum Society. [S0734-211X(00)01506-7]
\end{abstract}

\section{INTRODUCTION}

Interferometric displacement measuring systems have become commonplace in applications where nanometer resolution and large dynamic measurement range are required. Examples are diamond ruling and turning, integrated circuit photolithography, laser and electron beam lithography, and metrology at the nanometer level in general. Decreasing features sizes, increasing wafer sizes, and increasingly sophisticated lithography schemes continuously demand more accurate positioning and displacement measurement capabilities.

In displacement measuring interferometers the shift in the frequency of a light beam reflected from a mirror attached to a moving stage is used to determine the velocity of a substrate mounted to the stage, from which the displacement is simply derived through integration. Accuracy and precision of interferometric displacement measurements are compromised by a wealth of sources of error, such as alignment errors, polarization and frequency mixing, nonplanar wave fronts, lack of environmental control, and insufficient laser stability. ${ }^{1,2}$ Efforts are underway around the world to reduce these errors to the subnanometer level.

In our ongoing studies of errors that degrade interferometer performance we noticed that so far no attention has been paid to relativistic effects, presumably due to the comparatively small stage velocities involved. However, high sample stage velocities are desirable for higher throughput in volume manufacturing and can be on the order of meters per second. ${ }^{3}$ Since these velocities are still eight orders of magnitude smaller than the speed of light, relativistic effects are very small, but with attainable position resolutions at and below the nanometer level and displacements on the order of $1 \mathrm{~m}$ they can in general not be neglected anymore. For example a recently proposed method for the manufacturing of

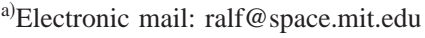

large-area, fine-period, and low-distortion gratings and grids requires the ability to repeatedly scan a single large wafer many times at high speed with nanometer accuracy under a $\mathrm{mm}^{2}$ sized interference pattern. ${ }^{4}$

In the following, we first review the principles underlying heterodyne interferometric displacement measurements. We then calculate the relativistic Doppler shift for the general case of a single reflection from a mirror that moves arbitrarily within a plane. Next we consider the case of a multiple-pass interferometer and the effects of varying optical path lengths due to the mirror motion. Relativistic corrections are discussed for realistic velocity profile examples.

\section{HETERODYNE INTERFEROMETERS}

A typical heterodyne interferometer setup is shown in Fig. 1. Laser light emitted with frequency $f_{0}$ is split into two orthogonally polarized beams of frequency $f_{0}$ and $f_{1}$ by Zeeman splitting or an acousto-optic modulator (AOM). ${ }^{5}$ Their frequency difference $F_{1}=f_{1}-f_{0}$ serves as a reference signal and can be obtained from phase meter PM1. A polarizing beam splitter reflects the beam with frequency $f_{0}$ to a stationary mirror or retroreflector, while the beam with frequency $f_{1}$ passes through to a mirror or retroreflector rigidly connected to a movable sample stage. After reflection by the respective mirrors both beams are recombined through the beam splitter and their phase difference is measured by phase meter PM2. Motion of the sample stage along the propagation direction of the beam with frequency $f_{1}$ ( $x$ direction) leads to a Doppler shift $\Delta F=f_{1}-f_{2}$ in the frequency $f_{2}$ of the reflected beam. Measurement of the frequency difference $F_{2}=f_{2}-f_{0}$ and comparison with the reference signal $F_{1}$ allows one to calculate $\Delta F$. The classical result for the Doppler shift is often presented in the form $\Delta F=2 M n v(t) / \lambda$, where $\lambda$ is the wavelength of light with frequency $f_{1}, n$ is the index of refraction of the medium along the light path 


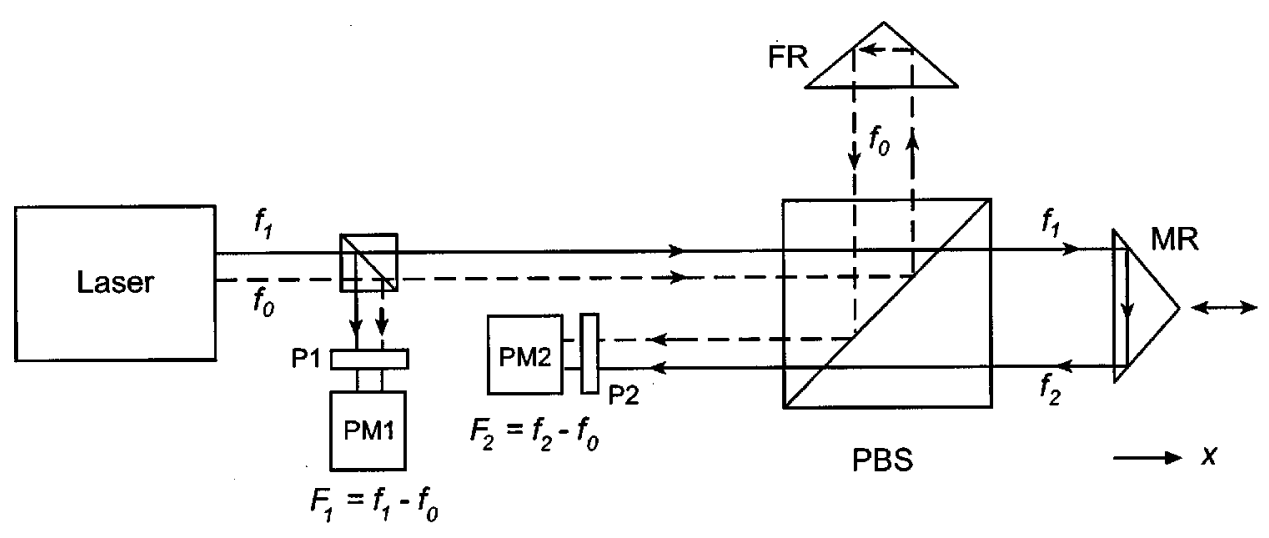

FIG. 1. Schematic of a typical heterodyne displacement measuring interferometer setup. The two overlapping laser beams with frequencies $f_{0}$ and $f_{1}$ have opposite polarization. Phase meter PM1 measures their frequency difference $F_{1}=f_{1}-f_{0}$ after they pass through mixing polarizer $\mathrm{P} 1$. The polarizing beam splitter (PBS) directs beam $f_{0}$ to the fixed retroreflector (FR) and beam $f_{1}$ to the moving retroreflector (MR). Phase meter PM2 measures the frequency difference between the reflected beams $F_{2}=f_{2}-f_{0}$ after they pass through mixing polarizer P2. (which we set equal to 1 henceforth), $M$ is the number of passes of the beam to the movable mirror, and $v(t)$ is the velocity of the stage along the light path at time $t$. If we assume, for example, $\lambda=633 \mathrm{~nm}$ and $v=1.0 \mathrm{~m} / \mathrm{s}$, we obtain $\Delta F=6.3 \mathrm{MHz}$ for two passes. In principle, by integrating the velocity over time the displacement of the stage can be determined to arbitrary accuracy, assuming a sufficiently small measurement time resolution and neglecting all other sources of error. Modern two-pass interferometers achieve a resolution of $\lambda / 2048=0.32 \mathrm{~nm}^{3}$

\section{RELATIVISTIC DOPPLER SHIFT}

In order to calculate the relativistic effects on the Doppler shift we generalize a previous approach ${ }^{6}$ to the problem of a mirror moving at an arbitrary velocity within two dimensions.

We consider the following idealized situation: A plane wave of light of frequency $f_{1}=\omega_{1} / 2 \pi$ is emitted at $x=0$ in the $x$ direction of the laboratory or rest frame. This light wave can be expressed as a four-dimensional wave vector

$$
k_{1}=\left(\begin{array}{c}
k_{t} \\
k_{x} \\
k_{y} \\
k_{z}
\end{array}\right)=\left(\begin{array}{c}
\omega_{1} / c \\
k_{x} \\
0 \\
0
\end{array}\right)
$$

$$
\Lambda_{1}=\left(\begin{array}{cccc}
\gamma & -\left(v_{x} / c\right) \gamma & -\left(v_{y} / c\right) \gamma & 0 \\
-\left(v_{x} / c\right) \gamma & 1+(\gamma-1) v_{x}^{2} / v^{2} & (\gamma-1) v_{x} v_{y} / v^{2} & 0 \\
-\left(v_{y} / c\right) \gamma & (\gamma-1) v_{x} v_{y} / v^{2} & 1+(\gamma-1) v_{y}^{2} / v^{2} & 0 \\
0 & 0 & 0 & 1
\end{array}\right)
$$

$$
\gamma=\frac{1}{\sqrt{1-v^{2} / c^{2}}},
$$

(relativistic aberration $\propto v_{y} / c$ ) relative to the mirror normal

$$
k_{1}^{\prime}=k_{x} \gamma\left(\begin{array}{c}
1-v_{x} / c \\
1 / \gamma-v_{x} / c+(1-1 / \gamma) v_{x}^{2} / v^{2} \\
-v_{y} / c+(1-1 / \gamma) v_{x} v_{y} / v^{2} \\
0
\end{array}\right)
$$

and $v^{2}=v_{x}^{2}+v_{y}^{2}$. In the moving frame of the mirror we thus have a Doppler-shifted beam incident at a small angle where $k_{t}=\omega_{1} / c=\left(k_{x}^{2}+k_{y}^{2}+k_{z}^{2}\right)^{1 / 2}$ describes the timelike component as the ratio between optical angular frequency $\omega_{1}$ and the speed of light in vacuum $c$. Since the beam propagates in the $x$ direction, the only spacelike component different from zero is the $x$ component with magnitude $k_{x}$ $=2 \pi / \lambda$. The beam is incident on a flat mirror, situated at some distance $x(t)$ and oriented with its normal in the negative $x$ direction. The mirror is moving at some velocity $\mathbf{v}(t)=\left[v_{x}(t), v_{y}(t)\right]$ relative to the rest frame. The incident beam is reflected back to $x=0$, where its frequency is measured in the rest frame. In order to calculate in the rest frame the relativistic frequency shift that the reflected beam has undergone we first transform the four-vector into the moving frame of the mirror. In that frame the beam is simply reflected specularly $\left(k_{x} \rightarrow-k_{x}\right)$. We then transform the fourvector of the reflected beam back into the rest frame to obtain its frequency shift observed by phase meter PM2.

The incident beam in the moving frame is calculated via

$$
k_{1}^{\prime}=\Lambda_{1} k_{1},
$$

where $\Lambda_{1}$ is the Lorentz transformation matrix given by 
Reflection in the moving frame simply results in a change in the sign of the $x$ component of $k_{1}^{\prime}$. The reflected beam $k_{2}^{\prime}$ is then transformed back into the rest frame through

$$
k_{2}=\Lambda_{2} k_{2}^{\prime}=\Lambda_{1}^{-1} k_{2}^{\prime} .
$$

The inverse Lorentz transformation matrix $\Lambda_{2}$ is simply obtained from $\Lambda_{1}$ by reversing the sign of all velocity components. After matrix multiplication and regrouping of terms we obtain for the timelike component of the reflected beam in the laboratory frame

$$
\omega_{2} / c=k_{x} \gamma^{2}\left[\left(1-v_{x} / c\right)^{2}+\frac{v_{y}^{2}}{c^{2}}\left(2 \frac{v_{x} c}{v^{2}}(1-1 / \gamma)-1\right)\right] .
$$

The factor $\gamma^{2}$ can be expanded in powers of $v / c$ :

$$
\gamma^{2}=1+\sum_{n=1}^{\infty}\left(\frac{v}{c}\right)^{2 n}
$$

The frequency is then given up to third order in $v_{x} / c$ by

$$
f_{2}=f_{1}\left[1-2 \frac{v_{x}}{c}+2\left(\frac{v_{x}}{c}\right)^{2}-\left(\frac{v_{x}}{c}\right)^{3}\left(2+\frac{v_{y}^{2}}{v_{x}^{2}}\right)+O\left(\frac{v_{x}}{c}\right)^{4}\right] .
$$

This result is interesting in a number of ways. First we see that the frequency depends on both velocity components of the moving mirror. The dependence on the velocity in the $y$ direction, however, is very weak and only enters in third order. Furthermore, this does not comprise a transverse Doppler shift in the rest frame, since the frequency only changes if $v_{x} \neq 0$. Most important is the difference between relativistic and classical calculations. In the classical case the Doppler-shifted frequency of the reflected beam is given by

$$
f_{2}=f_{1}\left(1-2 \frac{v_{x}}{c}\right)
$$

i.e., only the term of first order in $\left(v_{x} / c\right)$ is considered. In interferometric displacement measurements the $\left(v_{x} / c\right)^{2}$ term is at least eight orders of magnitude smaller than the leading velocity term even at relatively large stage velocities of a few meters per second. However, ignoring this term can introduce significant errors in certain situations discussed below. Essentially, neglecting the second-order velocity term leads to distance errors on the order of $v / c$, which can amount to several parts per billion, or several nanometers per meter at a velocity of $1 \mathrm{~m} / \mathrm{s}$.

Equation (3.10) can immediately be solved for $v_{x}$ and integrated to give the classical displacement with time

$$
x_{\mathrm{cl}}(t)=\int_{0}^{t} \frac{f_{1}-f_{2}\left(t^{\prime}\right)}{2 f_{1}} c d t^{\prime} .
$$

The actual measurement consists of readings of phase differences $\left\{\Delta \Phi_{i}\right\}$ at some sampling rate $1 / \Delta T$, which is typically on the order of $10^{7} \mathrm{~Hz}$. Frequency is related to phase by $f=(1 / 2 \pi) d \Phi / d t$, and the phase differences $\Delta \Phi_{i}$ are determined from $\Delta \Phi_{i}=\Phi_{1, i}-\Phi_{2, i}$, where $\Phi_{1, i}$ and $\Phi_{2, i}$ are the phase measurements of phase meters PM1 and PM2, respec- tively. The displacement deduced from the measurement is therefore more accurately described as a discrete sum over phase differences

$$
x_{\mathrm{cl}}(t)=\frac{c}{4 \pi f_{1}} \sum_{i=1}^{N} \Delta \Phi_{i}
$$

where $N=t / \Delta T$.

However, this approach neglects terms on the order of $\left(v_{x} / c\right)^{2}$ and higher and ignores the fact that the time it takes for the reflected, frequency shifted light to reach the phase meter is finite and depends on the variable distance between mirror and phase meter. In other words, the information on changes in the mirror velocity arrives at the detector delayed by a time $\delta t(t)=x(t) / c$, which leads to modifications on the same order as the relativistic Doppler shift corrections. Varying time delays also lead to variations in data-age, which can have detrimental effects when coordinating motion along multiple axes. ${ }^{3}$

\section{MULTIPASS INTERFEROMETERS AND OPTICAL PATH LENGTH VARIATIONS}

We now proceed to consider the general case of a beam reflected $M$ times off the movable mirror, and take into account the dependence of the optical path length as a function of the actual mirror displacement $x(t)$.

We begin by analyzing the situation for a double-pass interferometer $(M=2)$, where the beam is sent out to the mirror, reflected back to $x=0$, reflected out to the mirror for a second time, and finally sent back to the detector at the origin. Since at the end we will neglect terms of orders higher than $\left(v_{x} / c\right)^{2}$, we ignore motion of the mirror in the $y$ direction and therefore assume for simplicity $v=v_{x}$ [see Eq. (3.9)].

Let us consider light of frequency $f_{3}$ arriving at the detector at time $t$ after two reflections off the moving mirror. The second reflection took place at time $t_{2}=t-x\left(t_{2}\right) / c$, and the first reflection at time $t_{1}=t-2 x\left(t_{2}\right) / c-x\left(t_{1}\right) / c$. The final frequency is therefore given as

$$
f_{3}(t)=f_{1} \gamma^{2}\left[t_{1}\right]\left(1-\frac{v\left(t_{1}\right)}{c}\right)^{2} \gamma^{2}\left[t_{2}\right]\left(1-\frac{v\left(t_{2}\right)}{c}\right)^{2},
$$

where $\gamma^{2}[t]=\left[1-(v(t) / c)^{2}\right]^{-1}$. Since $t-t_{1}, t-t_{2} \ll t$, one can expand around $t$ :

$$
\begin{aligned}
f_{3}(t) \approx & f_{1}\left(\gamma^{2}[t]\left(1-\frac{v(t)}{c}\right)^{2}+\frac{2 a(t)\left[2 x\left(t_{2}\right)+x\left(t_{1}\right)\right]}{c^{2}[1+v(t) / c]^{2}}\right) \\
& \times\left(\gamma^{2}[t]\left(1-\frac{v(t)}{c}\right)^{2}+\frac{2 a(t) x\left(t_{2}\right)}{c^{2}[1+v(t) / c]^{2}}\right) \\
= & f_{1}\left(\gamma^{4}[t]\left(1-\frac{v(t)}{c}\right)^{4}+\gamma^{2}[t]\right. \\
& \left.\times\left(1-\frac{v(t)}{c}\right)^{2} \frac{2 a(t)\left[3 x\left(t_{2}\right)+x\left(t_{1}\right)\right]}{c^{2}[1+v(t) / c]^{2}}+O(1 / c)^{4}\right),
\end{aligned}
$$

where $a(t)=d v(t) / d t$. 
Expanding the $\gamma$ factors in powers of $(v / c)$ and neglecting terms of order $O(1 / c)^{3}$ one obtains

$$
\begin{aligned}
f_{3}(t) \approx & f_{1}\left(1-4\left(\frac{v(t)}{c}\right)+8\left(\frac{v(t)}{c}\right)^{2}\right. \\
& \left.+2\left(\frac{a(t)}{c^{2}}\right)\left[3 x\left(t_{2}\right)+x\left(t_{1}\right)\right]\right) .
\end{aligned}
$$

The last term is due to the changing displacement $x(t)$, while the other terms arise from the relativistic Doppler shift. To the same level of approximation we can further neglect the differences between the positions at times $t, t_{1}$, and $t_{2}$, resulting in

$$
f_{3}(t) \approx f_{1}\left(1-4\left(\frac{v(t)}{c}\right)+8\left[\left(\frac{v(t)}{c}\right)^{2}+\frac{a(t) x(t)}{c^{2}}\right]\right) .
$$

For the general case of $M$ reflections off the moving mirror the Doppler shift is given by

$$
f_{M+1}(t)=f_{1} \prod_{i=1}^{M} \gamma^{2}\left[t_{i}\right]\left(1-\frac{v\left(t_{i}\right)}{c}\right)^{2},
$$

and reflections take place at times

$$
t_{i}=t-x\left(t_{i}\right) / c-(2 / c) \sum_{j=i+1}^{M} x\left(t_{j}\right) .
$$

Following the same procedure as outlined for the case $M$ $=2$ leads to

$$
\begin{aligned}
f_{M+1}(t)= & f_{1}\left(1-2 M\left(\frac{v(t)}{c}\right)\right. \\
& \left.+2 M^{2}\left[\left(\frac{v(t)}{c}\right)^{2}+\frac{a(t) x(t)}{c^{2}}\right]+O\left(\frac{1}{c}\right)^{3}\right) .
\end{aligned}
$$

Again the classical result only takes the term of first order in $(v / c)$ into account:

$$
f_{M+1}=f_{1}\left(1-2 M \frac{v_{c l}(t)}{c}\right) \text {. }
$$

Before we proceed let us consider the situation where at $t=0$ the mirror starts from rest to move away from the detector and after some time returns and stops at the origin at time $t=T$. Since the detector/mirror system has returned to the same state (mirror at rest at $x=0$ ), the phase $\Phi_{M+1}$ at the detector at time $T$ has to be independent of what happened during the time interval $T$, i.e., it has to be the same as if the mirror had remained at rest for the whole time. More generally, this has to be true for any time interval $T$, where $x(0)$ $=x(T)$ and $v(0)=v(T)$. The phase $\Phi_{M+1}$ is obtained from integration of $f_{M+1}(t)$ :

$$
\Phi_{M+1}(T)=2 \pi \int_{0}^{T} f_{M+1}(t) d t=\Phi_{1}(T)=2 \pi f_{1} T,
$$

assuming that $\Phi_{1}(0)=\Phi_{M+1}(0), x(0)=x(T)$, and $v(0)$ $=v(T)$. This means that for any arbitrary velocity profile that returns the mirror to the same state every order of the velocity terms in Eq. (4.7) must independently integrate to zero. It is therefore intuitively clear that relativistic corrections to the displacement $x_{\mathrm{cl}}(t)$ derived from the classical Doppler formula cannot become arbitrarily large even when moving a mirror back and forth many times.

In all the current applications that we are aware of the measured frequency $f_{M+1}(t)$ serves to determine the velocity using the classical expression of Eq. (4.8). By equating Eqs. (4.7) and (4.8) this "classically" derived velocity $v_{\mathrm{cl}}$ is thus related to the actual velocity $v(t)$, up to terms of the order $(1 / c)$, by

$$
v_{\mathrm{cl}}(t) \approx v(t)-\frac{M}{c}\left(v^{2}(t)+a(t) x(t)\right) .
$$

Integrating $v_{c l}$ to obtain the displacement yields

$$
\begin{aligned}
x_{\mathrm{cl}}(t) & =\int_{0}^{t} v_{\mathrm{cl}}\left(t^{\prime}\right) d t^{\prime} \\
& \approx \int_{0}^{t}\left(v\left(t^{\prime}\right)-\frac{M}{c}\left[v^{2}\left(t^{\prime}\right)+a\left(t^{\prime}\right) x\left(t^{\prime}\right)\right]\right) d t^{\prime} \\
& =x(t)\left(1-M \frac{v(t)}{c}\right),
\end{aligned}
$$

assuming without loss of generality that $x(0)=0$. The lowest order error in the displacement is therefore proportional to the actual displacement, to the velocity at the time of the measurement, and to the number of reflections off the moving mirror. Referring back to Eqs. (3.11) and (3.12), the actual displacement can thus be determined from the phase difference readings $\left\{\Delta \Phi_{i}\right\}$ to a very good approximation via

$$
x(t) \approx \frac{c}{4 \pi f_{1} M}\left(\sum_{i=1}^{N} \Delta \Phi_{i}\right)\left(1+\frac{\Delta \Phi_{N}}{4 \pi f_{1} \Delta T}\right) .
$$

To minimize noise due to a single phase measurement it would of course be recommendable to replace $\Delta \Phi_{N}$ by the average of an appropriate number of most recent phase differences $\left(\Delta \Phi_{N} \longrightarrow(1 / L) \sum_{i=N-L+1}^{N} \Delta \Phi_{i}\right)$.

In the following we present some quantitative examples of expected errors in present and future applications.

In a typical step-and-scan photolithographic exposure tool a wafer is scanned in a serpentine pattern under the projection optics. Let us assume that the mask pattern is projected onto a wafer at fourfold reduction. This requires that the reticle stage moves in a highly synchronized manner with the wafer stage at four times the speed of the wafer. Let us further assume that the reticle stage has a trapezoidal velocity profile with a maximum speed of $1 \mathrm{~m} / \mathrm{s}$, and that it undergoes a maximum displacement of $0.25 \mathrm{~m}$. Figure 2 shows the error in the displacement, $x_{\mathrm{cl}}(t)-x(t)$, for the case of a two-pass interferometer. The maximum error is close to 1.3 $\mathrm{nm}$, which is several times the position resolution of stateof-the-art interferometers. However, upon fourfold image reduction the maximum pattern placement error will be only about $0.3 \mathrm{~nm}$. Both numbers are rather small compared to current minimum feature sizes with overlay budgets on the order of $25 \mathrm{~nm}$, and far below the precision presently achieved in synchronizing wafer and reticle stage movement. 


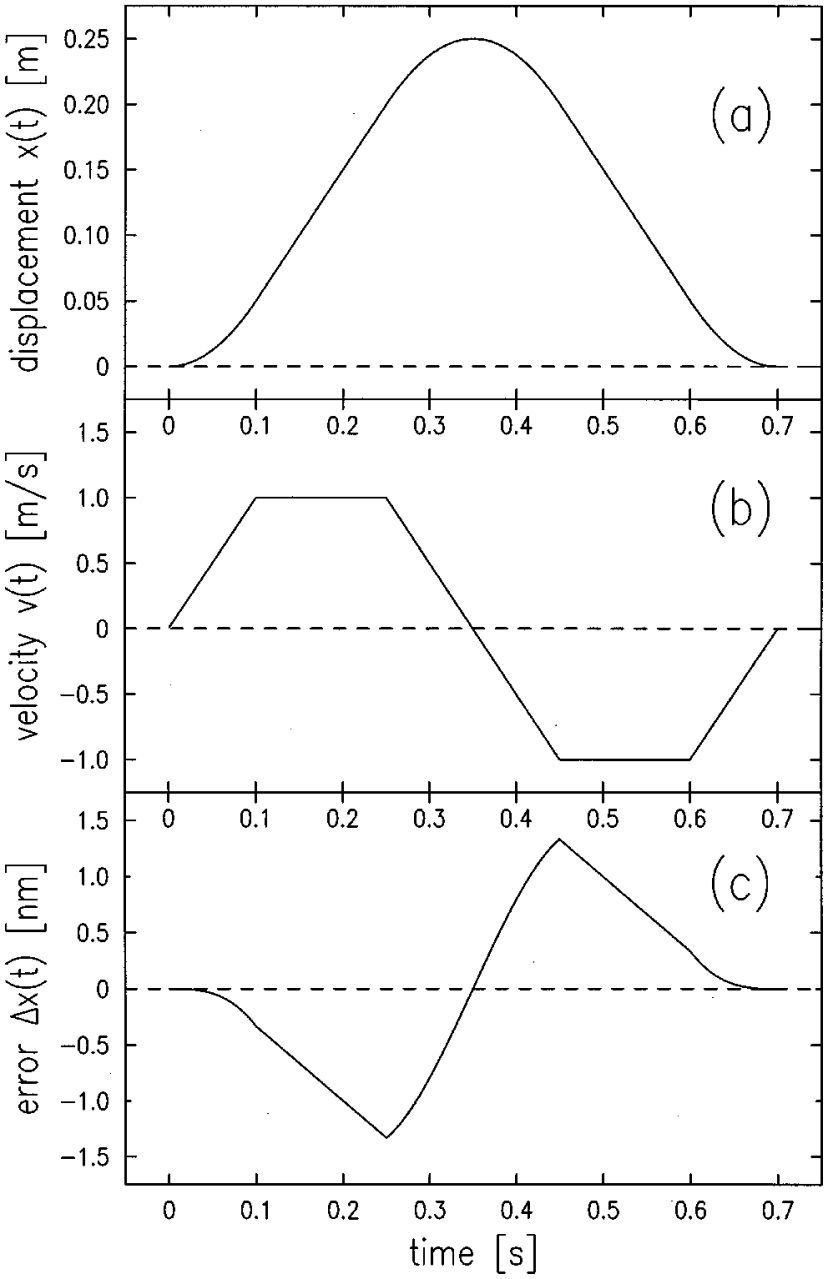

FIG. 2. (a) Model reticle stage displacement as a function of time for one scan back and forth across an exposure field compatible with a throughput of 90 wafers ( $200 \mathrm{~mm}$ diameter) per hour at fourfold image reduction between reticle and wafer. (b) Velocity profile corresponding to the displacement curve from (a). (c) Error in displacement, $x_{\mathrm{cl}}(t)-x(t)$, according to Eq. (4.11) for the velocity profile in (b) for a two-pass interferometer ( $M$ $=2)$.

An example where relativistic corrections are more important is scanning-beam interference lithography (SBIL), ${ }^{4}$ which is currently being developed in our laboratory. The challenging goal of this technique is the manufacture of large-area phase-coherent periodic patterns with subnanometer phase error for pattern-placement metrology and other applications. The main SBIL design idea is to overlap two laser beams in a $\mathrm{mm}^{2}$-sized area to obtain a small linear interference pattern with very low distortion. A substrate is then scanned through the interference pattern in a serpentine path to write large-area gratings.

To generate certain types of patterns it would be advantageous to scan the interference fringes through the overlap area synchronously with the substrate at high speed. To achieve this goal the displacement of the substrate must be known at all times with subnanometer accuracy. We desire the ability to scan back and forth over $300 \mathrm{~mm}$ at $1 \mathrm{~m} / \mathrm{s}$. In this case the displacement error according to Eq. (4.11) is $-2.0 \mathrm{~nm}$ at the far side of the wafer when moving away from the detector and $+2.0 \mathrm{~nm}$ on the way back $(M=2)$. The maximum stitching error between two neighboring exposure paths is therefore $4.0 \mathrm{~nm}$, more than twice our design goal and an order of magnitude greater than the resolution of the displacement measuring electronics.

Current state-of-the-art interferometer electronics are specified to achieve position resolution of $0.31 \mathrm{~nm}$ over a velocity range of $\pm 2.1 \mathrm{~m} / \mathrm{s}$ and a position range of $\pm 10.6 \mathrm{~m}$ for a two-pass system. ${ }^{3,7}$ Even though we are not aware of any present day applications that demand the full range of these specifications, moving only half the maximum position range at the maximum velocity would lead to considerable relativistic corrections of about $150 \mathrm{~nm}$.

In summary we have calculated first-order relativistic corrections in displacement measuring interferometry. For existing industrial applications as well as research applications currently under development those corrections can be on the order of nanometers and already exceed the position resolution of commercially available interferometer systems. Future increases in stage velocities and wafer sizes together with decreasing feature sizes that demand smaller pattern placement errors will require that relativistic corrections are properly taken into account. For all practical purposes the straightforward implementation of Eq. (4.12) in the electronics that convert phase measurements into displacement will completely remove relativistic errors from positioning and overlay error budgets.

\section{ACKNOWLEDGMENTS}

This work was supported by the Defense Advanced Research Projects Agency (DARPA) under Grant No. DAAG55-98-1-0130 and the National Aeronautics and Space Administration (NASA) under Grant No. NAG55105.

${ }^{1}$ N. Bobroff, Meas. Sci. Technol. 4, 907 (1993).

${ }^{2}$ E. C. Teague, in Technology of Proximal Probe Lithography, SPIE Institutes for Advanced Optical Technologies Series, edited by C. R. K. Marrian, IS-10 (SPIE, Bellingham, WA, 1993), pp. 322-363.

${ }^{3}$ F. C. Demarest, Meas. Sci. Technol. 9, 1024 (1998).

${ }^{4}$ M. L. Schattenburg, C. Chen, P. N. Everett, J. Ferrera, P. Konkola, and H. I. Smith, J. Vac. Sci. Technol. B 17, 2692 (1999).

${ }^{5}$ For more details, see for example, A. H. Slocum, Precision Machine Design (Prentice Hall, Englewood Cliffs, NJ, 1992).

${ }^{6} \mathrm{P}$. Hickson, R. Bhatia, and A. Iovino, Astron. Astrophys. 303, L37 (1995).

${ }^{7}$ ZMI-2001 Measurement Board P/N 8020-0210-01 Operating Manual OMP-0403E (1998); Zygo Corporation, Middlefield, CT 06455-0448. 